\title{
Editorial
}

\section{Mario Plebani \\ Biomarkers of acute kidney injury: a step forward}

DOI 10.1515/cclm-2017-0300

Acute kidney injury (AKI) reflects a broad spectrum of clinical presentations ranging from mild to severe injury that may result in dysfunction with recovery or nonrecovery, leading to some or permanent and complete loss of renal function that is associated with substantial morbidity, mortality and costs. The incidence of AKI is increasing because of increased patient's susceptibility (aging and comorbidities) and intensity of exposures (drugs, contrast media and major surgery). Despite advancements in diagnosis and care practice, AKI remains a disorder usually under/late recognized with high mortality [1]. One of the hidden reasons for persistent poor clinical outcomes may be the delay in timing for nephrology consultation as nephrologists are usually involved when severe AKI has already settled. According to the "iceberg of AKI" concept, many conditions are actually hidden under the cover of the tip of the iceberg although they should require the same or more attention by nephrologists but are usually unnoticed or self-managed by clinicians [2]. The Vicenza ADQI consensus conference group has introduced RIFLE criteria in 2004, allowing to make a standardized diagnosis of AKI and to define its clinical stage [3]. RIFLE classification allows characterizing AKI severity based on serum creatinine changes and urine output. Different RIFLE classes present remarkably different morbidity and mortality outcomes. The criteria have been subsequently modified leading to the current Kidney Disease Global Outcome Initiative (KDIGO) guidelines for diagnosis and care of AKI [4, 5]. Several lines of evidence, however, suggest that current diagnostic criteria are inadequate to characterize the continuum of the AKI syndrome from its initial phases to the advanced forms of kidney failure [6]. For this reason, a conceptual model of AKI has been developed encompassing all the steps of the syndrome and the relevant clinical consequences [5]. The RIFLE classification has made a significant progress [2-4], but it still relies on creatinine, precluding the possibility of a timely and accurate AKI diagnosis. Creatinine in fact is a poor marker for injury, and its elevation may occur 24-48 h after a significant insult to the kidney has occurred. Furthermore, its value may remain normal in case of subclinical forms of kidney dysfunction and damage. Over the last few years, several new AKI biomarkers have been discovered and validated to improve early detection, differential diagnosis and prognosis and better patient management (Figure 1). In addition, biomarkers may also be used to identify a status of kidney stress or an increased susceptibility to insults. In such conditions, they may trigger early preventive and protective measures well before AKI becomes manifest according to the KDIGO [7]. The utilization of biomarkers in AKI has been predicted to mirror what happened in the acute coronary syndrome in the absence of ST elevation on the electrocardiogram, where cardiac troponin assay made possible the diagnosis of non-ST elevation myocardial infarction [8]. However, although some new biomarkers have been studied and viewed as promising, no new biomarker has been universally accepted for routine use in clinical practice. Each of new biomarkers presents advantages and shortcomings (particularly related to the specificity for AKI), and more robust cost-effectiveness analyses should be performed before a wide clinical adoption. In this issue of the journal, Kashani and colleagues publish a very interesting review on biomarkers in AKI analyzing the pathway from discovery to clinical adoption [9].

The authors reviewed the available literature on characteristics of promising AKI biomarkers that are currently the focus of preclinical and clinical investigations. These biomarkers include neutrophil gelatinase-associated lipocalin, kidney injury molecule 1, liver-type fatty acidbinding protein, interleukin 18, insulin-like growth factorbinding protein 7 , tissue inhibitor of metalloproteinase 2, calprotectin, urine angiotensinogen and urine microRNA. The authors describe the clinical performances of these biomarkers for diagnosis and prognostication and appraise each AKI biomarker's advantages and limitations as a tool for early AKI recognition and prediction of clinical outcomes after AKI.

Some "take-home messages" of this paper should be highlighted. First, the roadmap toward a routine adoption of biomarkers has to include a thorough health technology assessment (HTA) and a well-defined cost-benefit analysis. HTA should consist of a multidisciplinary process that provides an appropriate platform for well-designed studies and analyses [10]. Second, clinical implementation of AKI biomarkers requires a multidisciplinary team approach 


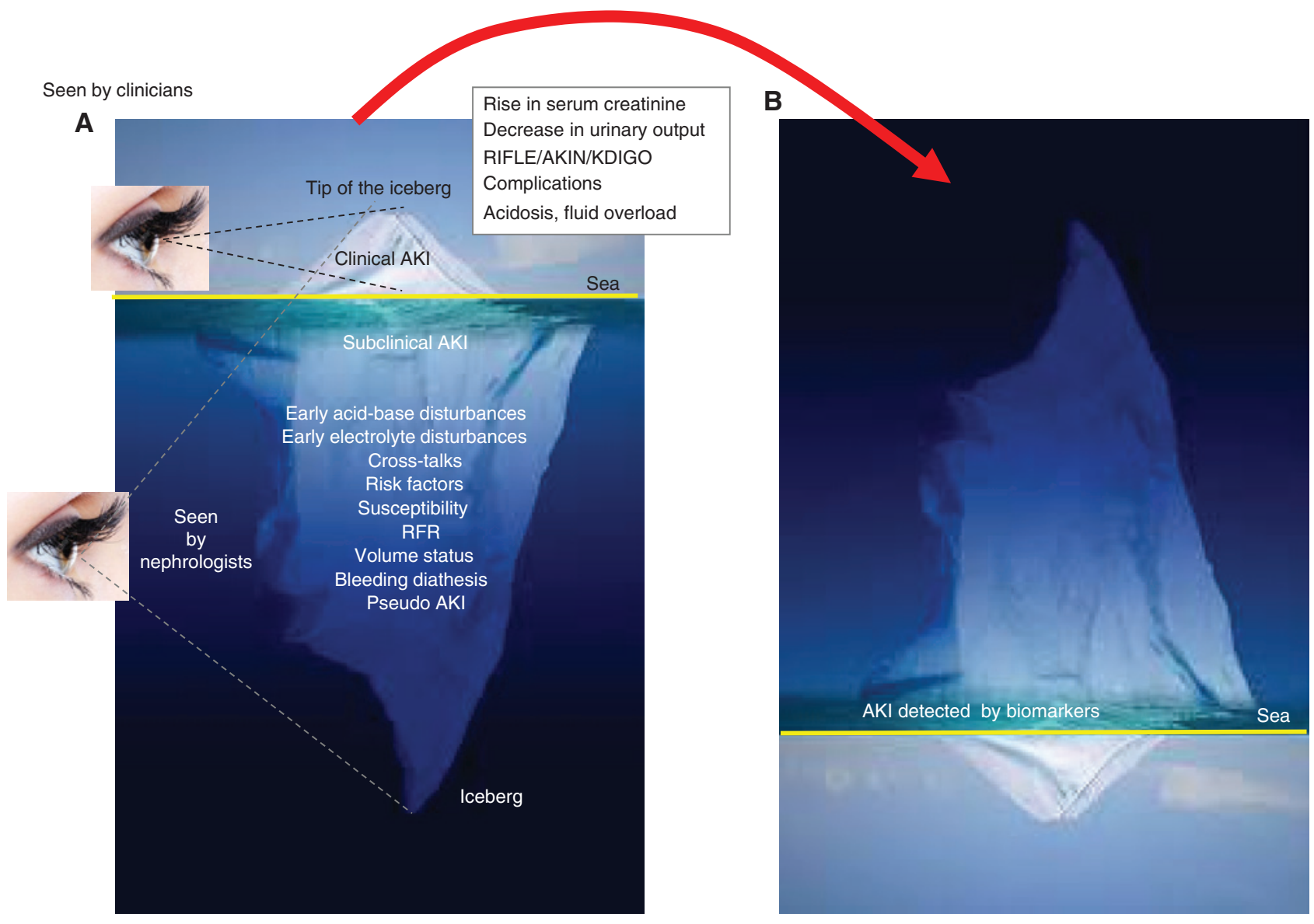

Figure 1: The iceberg of AKI.

Hidden conditions under the cover of the tip of the iceberg when traditional criteria are adopted (A). Improvement in AKI diagnosis by using biomarkers (B). From Reference 2, modified.

and a sound alliance among clinical providers and laboratory professionals to identify high-risk patients and "protocolized measurement" of biomarkers. Third, clinical prediction models for risk stratification of patients in the hospital or Intensive Care Units should be implemented via the advent of electronic surveillance tools that are able to identify high-risk patients. It was recently emphasized that the current biomarker pipeline is "too prone to failures" [11]. Improvements can include the use of more stringent methodology, better reporting, larger collaborative studies, careful external independent validation, rigorous systematic reviews and implementation studies. However, the starting point should be a careful consideration of clinical needs. In the case of biomarkers of AKI, the clinical needs are evident, but a common weak point is the perception of the lack of clinical utility due to little availability of effective therapeutic interventions once the early diagnosis is established. Current research should generate more data by adopting reliable and accurate biomarkers to identify risk factors and early signs of disease to trigger specific interventions potentially affecting the patient's clinical outcomes. By following this roadmap, it should be possible to avoid the dramatic failure of the biomarker pipeline defined as "lost in translation" [12]. Biomarkers may allow to make the diagnosis of subclinical AKI when classic criteria are still within normal range. Specific biomarkers may represent a molecular signature for a specific type of insult (for example ischemia, sepsis, toxic, etc.). Moving from clinical to molecular diagnosis of AKI may allow characterizing the causative role of specific pathogenic factors and may help to develop individual criteria and decision-making frameworks for the etiological variants of AKI. In conclusion, the field is in continuous evolution, and the curve of adoption seems to move forward from early research studies into more structured clinical utilization. This will require an integrated approach where the clinical and the laboratory medicine should find a common ground for a new strategic alliance.

Author contributions: The author has accepted responsibility for the entire content of this submitted manuscript and approved submission. 
Research funding: None declared.

Employment or leadership: None declared.

Honorarium: None declared.

\section{References}

1. Kellum JA, Bellomo R, Ronco C. Kidney attack. J Am Med Assoc 2012;307:2265-6.

2. Soares DM, Pessanha JF, Sharma A, Brocca A, Ronco C. Delayed nephrology consultation and high mortality on acute kidney injury: a meta-analysis. Blood Purif 2017;43:57-67.

3. Bellomo R, Ronco C, Kellum JA, Mehta RL, Palevsky P; Acute Dialysis Quality Initiative workgroup. Acute renal failure definition, outcome measures, animal models, fluid therapy and information technology needs: the Second International Consensus Conference of the Acute Dialysis Quality Initiative (ADQI) Group. Crit Care 2004;8:R204-12.

4. Kellum JA, Mehta RL, Levin A, Molitoris BA, Warnock DG, Shah SV, et al. Development of a clinical research agenda for acute kidney injury using an international, interdisciplinary, three-step modified Delphi process. Clin J Am Soc Nephrol 2008;3:887-94.

5. Kellum JA, Lameire N; KDIGO AKI Guideline Work Group. Diagnosis, evaluation, and management of acute kidney injury: a KDIGO summary (Part 1). Crit Care 2013;17:204-7.

6. de Geus HR, Ronco C, Haase M, Jacob L, Lewington A, Vincent JL. The cardiac surgery-associated neutrophil gelatinase-associated lipocalin (CSA-NGAL) score: a potential tool to monitor acute tubular damage. J Thorac Cardiovasc Surg 2016;151:1476-81.
7. Katz N, Ronco C. Acute kidney stress-a useful term based on evolution in the understanding of acute kidney injury. Crit Care 2016;20:23-6.

8. Roffi M, Patrono C, Collet JP, Mueller C, Valgimigli M, Andreotti $\mathrm{F}$, et al. Management of acute coronary syndromes in patients presenting without persistent ST-segment elevation of the european society of cardiology. 2015 ESC Guidelines for the management of acute coronary syndromes in patients presenting without persistent ST-segment elevation: task force for the management of acute coronary syndromes in patients presenting without persistent ST-segment Elevation of the European Society of Cardiology (ESC). Eur Heart J 2016;37:267-315.

9. Kashani K, Cheungpasitporn W, Ronco C. Biomarkers of acute kidney injury: the pathway from discovery to clinical adoption. Clin Chem Lab Med 2017;55:1074-89.

10. Liguori G, Belfiore P, D'Amora M, Liguori R, Plebani $M$. The principles of health technology assessment in laboratory medicine. Clin Chem Lab Med 2017;55:32-7.

11. Ioannidis JP, Bossuyt PM. Waste, leaks, and failures in the biomarker pipeline. Clin Chem 2017;63:963-72.

12. Plebani M, Marincola FM. Research translation: a new frontier for clinical laboratories. Clin Chem Lab Med 2006;44:1303-12.

Corresponding author: Mario Plebani, CCLM Editor-in-Chief, Department of Laboratory Medicine, University-Hospital of Padua, Via Giustiniani 2, 35128, Padua, Italy, Phone: +390498212792 , Fax: +39 049663240, E-mail: mario.plebani@unipd.it. http://orcid.org/0000-0002-0270-1711 